Running head: Prior familiarity enhances unconscious learning

\title{
PRIOR FAMILIARITY WITH COMPONENTS ENHANCES UNCONSCIOUS LEARNING OF RELATIONS
}

\author{
Ryan B. Scott and Zoltan Dienes \\ Department of Psychology \\ University of Sussex
}

Ryan Scott

Department of Psychology,

Pevensey Building,

University of Sussex,

Falmer,

BN1 9QH

Telephone: +44(0)1273876638

Fax: +44 (0)1273 678058

Email: r.b.scott@sussex.ac.uk 


\begin{abstract}
The influence of prior familiarity with components on the implicit learning of relations was examined using artificial grammar learning. Prior to training on grammar strings, participants were familiarised with either the novel symbols used to construct the strings or with irrelevant geometric shapes. Participants familiarised with the relevant symbols showed greater accuracy when judging the correctness of new grammar strings. Familiarity with elemental components did not increase conscious awareness of the basis for discriminations (structural knowledge) but increased accuracy even in its absence. The subjective familiarity of test strings predicted grammaticality judgments. However, prior exposure to relevant symbols did not increase overall test string familiarity or reliance on familiarity when making grammaticality judgments. Familiarity with the symbols increased the learning of relations between them (bigrams and trigrams) thus resulting in greater familiarity for grammatical versus ungrammatical strings. The results have important implications for models of implicit learning.
\end{abstract}

Keywords or phrases:

Familiarity

Implicit learning

Artificial grammar learning

Unconscious knowledge

Perceptual learning

Associative learning 


\section{Introduction}

Much of learning consists of acquiring knowledge of relations or associations between things. In learning languages for example, we acquire knowledge of the relations between different types of word that occur in valid clauses. Knowledge of those relations need not necessarily be conscious - we can recognise grammatical errors without consciously knowing the relations that have been violated. Different models of learning make diverse predictions regarding how the learning of relations will be affected by prior familiarity with the components of those relations. Predictions range from learning being enhanced by greater familiarity with the components (Anderson, 1983), through learning being unaffected by the extent of familiarity (McClelland \& Rumelhart, 1986), to learning being actively inhibited by prior exposure to the component elements (Lubow \& Moore, 1959). In the present study we evaluate the influence of prior familiarity on the implicit learning of relations. Employing the artificial grammar learning (AGL) paradigm, we examine whether familiarity with individual components enhances the acquisition of unconscious knowledge regarding their relations.

AGL has proved a useful paradigm for the investigation of implicit learning (Pothos, 2007; Reber, 1989). In a typical AGL experiment participants are exposed to strings of letters or symbols generated by a finite state grammar, see Figure 1. The grammar imposes structural rules on the order of symbols making up the strings e.g. constraining which symbols can precede which others. The strings are typically presented under the guise of a short-term memory task with participants unaware of these rule-based constraints. At test, after being informed of the existence of rules, but not their precise nature, participants judge which of a new set of strings are grammatical. Participants typically discriminate grammatical strings with above-chance accuracy despite being unable to verbalise the nature of what has been learnt (e.g. Reber, 1989). Reber originally proposed that the ability to discriminate grammatical strings resulted from the implicit acquisition of regularities 
encountered during learning. Research has since shown these regularities to include commonly recurring fragments or chunks of the training strings (Dulany, Carlson, \& Dewey, 1984; Knowlton \& Squire, 1994; Perruchet \& Pacteau, 1990; Servan Schreiber \& Anderson, 1990), the pattern of repetitions within training strings (Brooks \& Vokey, 1991; Vokey \& Higham, 2005), and knowledge of whole training exemplars (Vokey \& Brooks, 1992). Acquiring sensitivity to any of these regularities entails learning at least a subset of the relations between the symbols making up the strings.

Servan-Schreiber and Anderson (1990) proposed that the similarity between training and test strings arising from fragmentary knowledge may result in differential feelings of familiarity (see Dienes, Scott, \& Wan, in press, for a review of the role of familiarity in implicit learning). The resulting familiarity account holds that grammatical strings, by virtue of conforming to the grammar, are more likely to have properties seen in training and will consequently feel more familiar. Discrimination performance then results from more familiar strings being endorsed as grammatical. There is now considerable evidence supporting this account. For example, the objective similarity of training and test strings strongly predicts grammaticality judgments (e.g. Johnstone \& Shanks, 2001). Signal detection analyses of implicit learning tasks are also consistent with decisions based on a continuous underlying dimension, such as familiarity, but not with certain rule-based accounts e.g. where a limited number of rules lead to black and white decisions (Kinder \& Assmann, 2000; Lotz \& Kinder, 2006). And most recently, Scott and Dienes (2008) showed that after incidental learning of an artificial grammar participants' subjective ratings of whole test-string familiarity reliably predicted their grammaticality judgments. The feelings of familiarity themselves have also been shown to derive primarily from the structural similarity of the training and test strings and not from differences in processing fluency (Scott \& Dienes, in press-a). 
While participants make judgments which reflect knowledge of the regularities in the test strings, often derived from familiarity, they at times lack conscious awareness of the knowledge they possess. There are at least two types of knowledge acquired in AGL which can be either conscious or unconscious; judgment knowledge and structural knowledge (Dienes \& Scott, 2005). Judgment knowledge relates to knowing whether or not a string is grammatical, while structural knowledge relates to knowing why a string is or is not grammatical e.g. knowing that a string is ungrammatical because of the presence of a particular combination of letters. The conscious status of judgment knowledge is typically assessed by the guessing criterion (Cheesman \& Merikle, 1986) or the zero-correlation criterion (Dienes, Altmann, Kwan, \& Goode, 1995). Where there is above chance accuracy, these measures hold there to be unconscious judgment knowledge if participants believe they are guessing, or if there is no relationship between their confidence and accuracy. Numerous studies have found AGL to result in unconscious judgment knowledge as assessed in this way (Allwood, Granhag, \& Johansson, 2000; Channon et al., 2002; Dienes \& Altmann, 1997; Dienes, Altmann, Kwan, \& Goode, 1995; Dienes \& Perner, 2003; Tunney \& Altmann, 2001). The presence of conscious judgment knowledge does not, however, imply the presence of conscious structural knowledge - knowledge of the relations between elements that permit the judgments. Participants may have confidence in a judgment based on a hunch or an overall feeling of familiarity for the test string without knowing what aspects of the string resulted in those feelings. Dienes and Scott (2005) introduced a simple methodology for assessing the conscious status of structural knowledge. Participants are asked to report the basis on which they made each judgment e.g. random selection, intuition, familiarity, rules, or recollection (where responses attributed to familiarity are defined as those based on the overall feeling of familiarity for a string without knowing which particular aspects make it familiar or unfamiliar). There is taken to be unconscious structural knowledge where there is 
above chance classification accuracy in responses attributed to random selection, intuition, or familiarity, and to be conscious structural knowledge in those attributed to rules or recollection. Dienes and Scott (2005) and Scott and Dienes (2008) demonstrate clear behaviour dissociations based on these distinctions.

The conscious status of judgment knowledge has been shown to be influenced by knowledge of the distribution of familiarity among test-strings. Scott and Dienes (2008; in press-b) showed that an extended test phase, enabling participants to learn the distribution of familiarity, increased their awareness of the knowledge they possessed without increasing their accuracy. It is thus apparent that whole-string familiarity, and knowledge of its distribution, has a central role in the development and conscious status of judgment knowledge. However, it has yet to be established whether prior familiarity with the individual components influences the acquisition or conscious status of the underlying structural knowledge i.e. of the relations between string components. In an investigation of the mereexposure effect, Zizak and Reber (2004) conducted AGL experiments where strings were comprised of either familiar or unfamiliar elements. The pattern of results was suggestive of performance being slightly better where familiar elements were used. However, as no statistical comparisons were conducted between the experiments no firm conclusions can be drawn.

Here we directly examine the influence of prior familiarity with components of an artificial grammar on the implicit learning of their relations and the conscious status of the knowledge acquired. For the first time performance is contrasted for participants either given or not given prior exposure to the novel symbols from which the strings are constructed. It was predicted that prior exposure would enhance the learning of relations between symbols (bigrams and trigrams) and that this would increase the relative familiarity of grammatical strings and hence the accuracy of classifications. However, no predictions were made 
regarding whether prior familiarity would influence the conscious status of the knowledge acquired. The findings are replicated in a second experiment and the results of both experiments presented together. ${ }^{1}$

\section{Method}

\section{Participants}

Participants were University of Sussex students participating in exchange for course credits. All participants were naive to the experimental hypothesis. Experiment 1 employed 40 participants (10 male and 30 female) with a mean age of $21(S D=2.5)$. Experiment 2 employed 64 participants (9 male and 55 female) with a mean age of $20(S D=2.8)$.

\section{Materials}

Two finite state grammars were used to generate grammar strings between seven and nine characters in length. Both grammars were taken from Reber (1969) with the usual letters replaced by novel symbols, see Figure 1. Both grammars use the same symbol-set and contain the same set of valid starting bigrams and final symbols. Training sets comprised sixteen strings (from the appropriate grammar) repeated three times in random orders. The test set comprised a combination of sixteen strings from each grammar (not seen in training) presented twice in random order. There was the same number of strings of each length in both training sets and the proportion of strings of each length was the same for training and test sets. The symbol strings were presented in black on a white background at the centre of a 12-in computer screen.

\section{Procedure}

Symbol Familiarisation. A perceptual clarification task was employed to familiarise participants with either relevant symbols (the five novel symbols used to construct the grammar strings) or irrelevant symbols (geometric shapes), see Figure 1. There were 100 randomly ordered trials with each symbol appearing 20 times. On each trial one of the five 
symbols gradually emerged from behind a masking rectangle at the centre of the screen. Participants were advised to press the space bar as soon as they could identify the symbol. When the spacebar was pressed the emerging symbol was removed and the participant indicated which symbol had appeared from a numbered list of the 5 possible answers. The fastest correct response was displayed in the corner of the screen to incentivise fast and accurate responding.

Training stage. Participants were advised that they were to memorise each of a sequence of forty-eight strings of symbols. Participants saw each string for 5 seconds followed by a blank screen for 5 seconds. Participants were supervised and required to write down what they could remember of each string while the blank screen was present but not before. The presentation order of both training and test strings was separately randomised for each participant.

Test stage. Participants were informed that the order of symbols in the training strings had obeyed a complex set of rules, and that they were to classify a new set of strings exactly half of which would obey the same rules; they were not told the rules themselves. Participants were asked: (1) to rate how familiar the string felt to them on a scale from $0-100(0=$ not at all familiar, $100=$ completely familiar); (2) to judge the string's grammaticality (obeyed the rules, yes or no); (3) to rate their confidence in their judgment on a scale from 50-100 (where $50=$ a guess, $100=$ certain); and (4) to indicate what they believed to be the basis for their grammaticality judgment (random selection, intuition, familiarity, rules, or recollection). These decision strategies were defined as follows: Random Selection - you had no confidence and literally chose yes or no at random; Intuition - you had some confidence but could not say on what you based your answer; Familiarity - you chose yes or no based on how familiar or unfamiliar the string felt but could not say why it was familiar or unfamiliar; Rules - you based your answer on one or more rules or partial rules that you derived and you 
could state the nature of the rules if required; Recollection - if you answered 'Yes' it was because you specifically recall encountering a sequence of symbols in training that resembled part or all of the present string. If you answered 'No' it was because you are certain that you didn't encounter any stimuli in training resembling the current one.

Design

The two grammar design of Dienes and Altmann (1997) was employed with half the participants trained on grammar A and half on grammar B. At test all participants classified the same test strings, exactly half of which conformed to each grammar. In this way the nongrammatical test strings for one group were grammatical for the other group thus eliminating the need for an untrained control group. The key independent variables were: (1) familiarisation condition, between subject (relevant symbols vs. irrelevant symbols), (2) grammaticality, within subject (grammatical vs. ungrammatical), and (3) decision strategy, within subject (random selection vs. intuition, vs. familiarity vs. rules vs. recollection). The main dependent variables of interest were (a) grammaticality judgment, and (b) subjective familiarity rating. An alpha level of .05 was used for all statistical tests and all reported $p$ values are for two-tailed significance. Effect sizes are provided in the form of Cohen's $d$ for difference scores and $r$ for correlations (Cohen, 1988).

\section{Results}

Consistent with predictions, participants who familiarised with the symbols making up the strings (relevant symbols) before training made significantly more correct grammaticality judgments $(M=66 \%, S E=1.3)$, than those who familiarised with the irrelevant geometric shapes, $(M=60 \%, S E=1.2), t(102)=3.22, p=.002, d=.66$. Greater familiarity with the symbols making up the strings facilitated learning the relations between symbols and hence the ability to discriminate grammatical from ungrammatical strings. 
The increased knowledge did not however result in greater conscious awareness of the basis for discrimination. ANOVA examining the percentage of responses attributed to the different decision strategies revealed no main effect or interaction involving familiarity condition (All $F<1$ ). Furthermore, the proportion of responses attributed to strategies indicating conscious knowledge of the relations (rules and recollection) was not significantly different for participants familiarising with relevant $(M=27 \%, S E=3.0)$ versus irrelevant symbols $(M=26 \%, S E=3.2), t(102)=.386, p=.700, d=.04, C I_{.95}=-10.5,+7.1$. Greater accuracy after familiarising with relevant versus irrelevant symbols was observed both for responses attributed to decision strategies indicating conscious structural knowledge (e.g. rules), and those indicating unconscious structural knowledge (e.g. intuition), see Figure 2. For responses attributed to intuition and familiarity, this indicates that prior familiarity with the components (the symbols) increased unconscious knowledge of the relations between them.

Consistent with previous research, the rated feelings of familiarity elicited by the test strings significantly predicted grammaticality judgments; more familiar strings were more likely to be endorsed as grammatical (Mean $r=.72, S E=.02), t(103)=43.99, p<.001$. This relationship did not differ significantly depending on whether participants had prior familiarity with relevant symbols $($ Mean $r=.74, S E=.02)$, or irrelevant symbols (Mean $r=$ $.70, S E=.03), t(102)=1.15, p=.254, d=.24, C I_{.95}=-.1,+.03$. The mean familiarity of test strings similarly did not differ depending on whether participants had familiarised with relevant $(M=47, S E=1.1)$ or irrelevant symbols $(M=45, S E=1.4), t(102)=1.11, p=.269$, $d=.22, C I_{.95}=-5.5,+1.5$. Crucially however, prior familiarity with relevant symbols increased the relationship between familiarity and grammatical status. The greater familiarity observed for grammatical over ungrammatical strings was more substantial after familiarising with relevant symbols $($ Mean $r=.35, S E=.02)$ than irrelevant symbols $($ Mean $r=.26, S E=$ 
$.02), t(102)=2.87, p=.005, d=.53$. As predicted, familiarity with the individual symbols prior to training enhanced the degree to which the relations between symbols became familiar.

The influence of prior familiarity on the learning of relations is also apparent in the correlation between familiarity ratings and the mean frequency with which bigrams and trigrams contained in test strings appeared during training (the associative chunk strength, Knowlton \& Squire, 1996). The relationship between familiarity and associative chunk strength was reliably greater for participants familiarised with relevant symbols (Mean $r=$ $.42, S E=.02)$ than irrelevant symbols $($ Mean $r=.33, S E=.02), t(102)=2.82, p=.006, d=$ .56. Where participants had prior familiarity with the individual symbols they more readily learnt the relations between symbols (e.g. bigrams and trigrams) and this resulted in greater familiarity for strings exhibiting more of those relations; typically grammatical strings.

\section{Discussion}

This study examined the effect that prior familiarity with the components used to instantiate an artificial grammar had on the extent of learning and the conscious status of the knowledge acquired. The results, replicated in both experiments, revealed that where novel symbols were used to construct grammar strings, prior familiarisation with those symbols increased learning. The increased learning was apparent in the accuracy of grammaticality judgments which, consistent with previous research, was related to the subjective familiarity of test strings; more familiar test strings were more likely to be endorsed as grammatical. Participants who familiarised with the symbols before learning were no more reliant upon familiarity in their judgments. However, the feelings of familiarity they experienced were more strongly related to the structural similarity of training and test strings. Specifically, familiarity was more strongly related to the frequency with which bigrams and trigrams appearing in test strings had occurred during training. Prior familiarity with the individual 
symbols appears to increase the ability of participants to learn the relations between them and results in greater feelings of familiarity when those same relations are encountered at test. While participants' knowledge of the relational consistencies of the grammar increased, there was no evidence that prior familiarity with the individual elements affected the conscious status of that knowledge (cf. Destrebecqz \& Cleeremans, 2003). Prior familiarity resulted in greater accuracy both for responses attributed to strategies indicating conscious structural knowledge (e.g. rules), and those indicating its absence (e.g. intuition). Prior familiarity with the elemental components thus enhanced both conscious and unconscious learning of the relations inherent in the grammar.

The increase in learning observed after familiarising with the individual components is consistent with the predictions of both the ACT memory model (Anderson, 1983) and McLaren and Macintosh's (2000) associative theory of perceptual learning. The ACT memory model holds that the strength of any concept will increase with practice and that the activation emitted by a concept is a function of its strength. As a consequence, new associations with familiar concepts will be acquired more rapidly than with unfamiliar concepts. Anderson demonstrated these effects in the context of explicit learning e.g. by showing that subjects retrieve new facts learnt about familiar people more rapidly than those learnt about less familiar people (e.g. Anderson, 1981). McLaren and Macintosh (2000) provide a detailed learning model that makes similar predictions based upon an elemental theory of latent inhibition. The standard phenomenon of latent inhibition is that prior exposure to individual stimuli inhibits the learning of associations between them (Lubow \& Moore, 1959). However, McLaren and Mackintosh demonstrate that where the component stimuli are themselves complex, then latent inhibition can facilitate perceptual learning. Numerous animal learning studies provide support for this hypothesis with associations involving one component of complex stimuli learned more quickly and generalising less after 
pre-exposure to another component (e.g. Darby \& Pearce, 1997; Mackintosh, Kaye, \& Bennett, 1991; Navarro, Hallam, Matzel, \& Miller, 1989). McLaren and Macintosh detail how a standard learning rule (the Delta rule) can be modified to implement this behaviour. The present findings highlight a weakness in current connectionist models of implicit learning (for a review see, Cleeremans \& Dienes, 2008). These models treat the individual components of an input vector - say the elements of a grammar string - in precisely the same way regardless of the number of times those inputs have occurred. As such, the models are insensitive to the effects of prior familiarity. The degree to which the relation between two symbols is learnt will be unaffected by the frequency with which the individual symbols have been encountered previously. In contexts where the components are already uniformly familiar the effect of this limitation may be negligible. However, the current results clearly indicate that to be capable of greater generalisation the models will require amendment. Implementing the modified learning rule proposed by McLaren and Mackintosh (2000) is one way this might be achieved. 


\section{Footnotes}

1. All the significant effects and null results reported collapsing the experiments together were also apparent when examining the experiments individually, with just one minor exception: the greater accuracy observed in the intuition, familiarity, and rules attributions after familiarising with relevant symbols (see Figure 2) was observed in Experiment 1, but was only achieved in the intuition and rules attributions in Experiment 2. A similar difference was observed for the familiarity attributions in Experiment $2(M=68 \%, S E=3.1$ vs. $M=$ $61 \%, S E=4.0$ ) but the difference did not achieve significance, $t(59)=1.37, p=.177, d=.36$. 


\section{Figure Captions}

Figure 1. An illustration of the experimental procedure including the finite state grammars and symbols employed.

Figure 2. Percentage correct by reported decision strategy (with SE), compared for familiarisation with relevant versus irrelevant symbols. 
Figures

Figure 1

\section{Familiarisation}

With Relevant Symbols

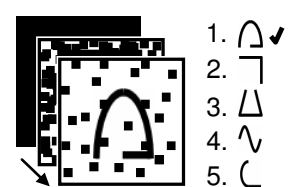

Or Irrelevant Symbols

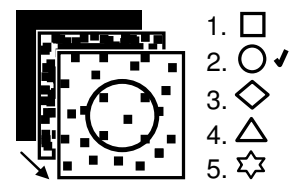

Grammar Training

On Grammar A strings

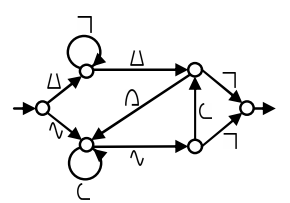

$\triangle \neg \neg \Delta\urcorner$

$\vee<v<7$

Or Grammar B strings

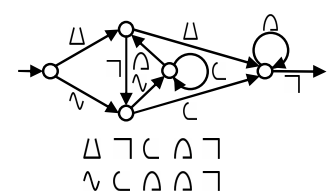

\section{Test Phase}

Classify new grammar strings from both grammar $A$ and $B$

1. $\triangle \neg C \triangle \triangle \neg$

2. $\triangle \neg \neg \Delta \triangle \vee \neg$

64. $\downarrow\llcorner\triangle \Delta ᄀ$

How familiar does it feel? (0-100) Does it obey the rules? (yes/no)

How confident are you? (50-100)

The basis for your judgment?

- Random selection

- Intuition

- Familiarity

- Rules

- Recollection 
Figure 2

\section{- Irrelevant Symbol Familiarisation}

\section{- Relevant Symbol Familiarisation}

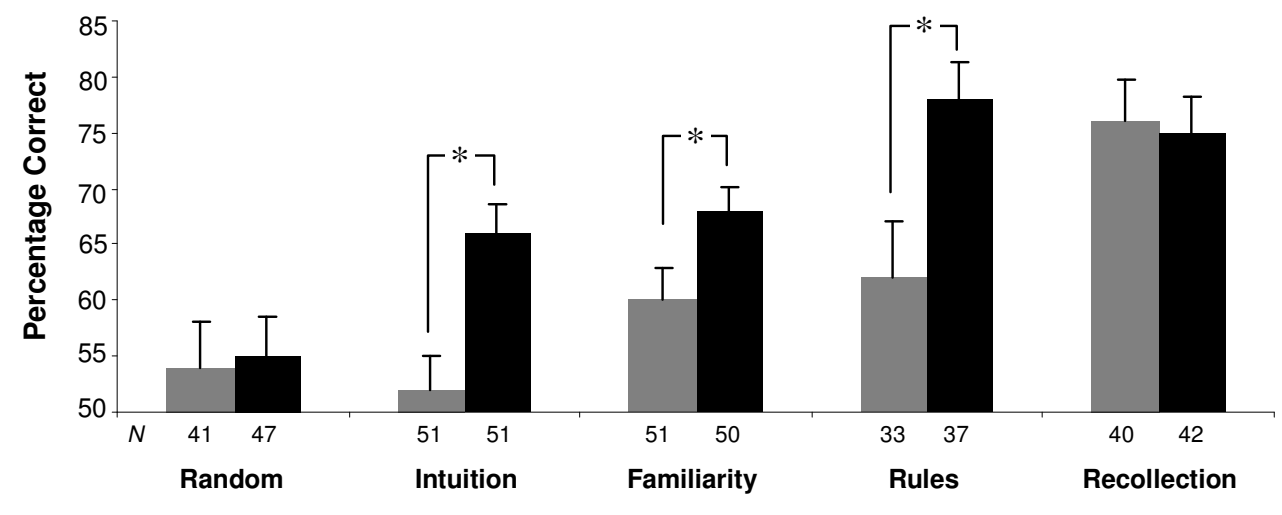

${ }^{*} p<.05$ 


\section{References}

Allwood, C. M., Granhag, P. A., \& Johansson, H. (2000). Realism in confidence judgements of performance based on implicit learning. European Journal of Cognitive Psychology, 12(2), 165-188.

Anderson, J. R. (1981). Effects of prior knowledge on memory for new information. Memory \& Cognition, 9, 237-246.

Anderson, J. R. (1983). The architecture of cognition. Cambridge, MA: Harvard University Press.

Brooks, L. R., \& Vokey, J. R. (1991). Abstract analogies and abstracted grammars: Comments on Reber (1989) and Mathews et al. (1989). Journal of Experimental Psychology: General, 120(3), 316-323.

Channon, S., Shanks, D. R., Johnstone, T., Vakili, K., Chin, J., \& Sinclair, E. (2002). Is implicit learning spared in amnesia? Rule abstraction and item familiarity in artificial grammar learning. Neuropsychologia, 40(12), 2185-2197.

Cheesman, J., \& Merikle, P. M. (1986). Distinguishing conscious from unconscious perceptual processes. Canadian Journal of Psychology, 40(4), 343-367.

Cleeremans, A., \& Dienes, Z. (2008). Computational models of implicit learning. In R. Sun (Ed.), Handbook of Computational Cognitive Modeling (pp. 396 - 421). Cambridge: University Press.

Cohen, J. (1988). Statistical power analysis for the behavioral sciences (2nd ed.). Hillsdale, NJ: Lawrence Earlbaum Associates.

Darby, R. J., \& Pearce, J. M. (1997). The effect of stimulus preexposure on responding during a compound stimulus. Quarterly Journal of Experimental Psychology Section B-Comparative and Physiological Psychology, 50(3), 203-216. 
Destrebecqz, A., \& Cleeremans, A. (2003). Temporal effects in sequence learning. In L. Jimenez (Ed.), Attention and implicit learning (pp. 181-213). Amsterdam, Netherlands: John Benjamins Publishing Company.

Dienes, Z., \& Altmann, G. (1997). Transfer of implicit knowledge across domains: How implicit and how abstract? In D. C. Berry (Ed.), How implicit is implicit learning? (pp. 107-123). London: Oxford University Press.

Dienes, Z., Altmann, G. T. M., Kwan, L., \& Goode, A. (1995). Unconscious knowledge of artificial grammars is applied strategically. Journal of Experimental Psychology: Learning, Memory, and Cognition, 21(5), 1322-1338.

Dienes, Z., \& Perner, J. (2003). Unifying consciousness with explicit knowledge. In A. Cleeremans (Ed.), The unity of consciousness: Binding, integration, and dissociation (pp. 214-232). New York, NY: Oxford University Press.

Dienes, Z., \& Scott, R. B. (2005). Measuring unconscious knowledge: distinguishing structural knowledge and judgment knowledge. Psychological Research, 69(5-6), $338-351$.

Dienes, Z., Scott, R. B., \& Wan, L. L. (in press). The role of familiarity in implicit learning. Essay in honour of Bruce Whittlesea. To appear. In P. Higham \& J. Leboe (Eds.), Constructions of Remembering and Metacognition.: Palgrave Macmillan.

Dulany, D. E., Carlson, R. A., \& Dewey, G. I. (1984). A case of syntactical learning and judgment: How conscious and how abstract? Journal of Experimental Psychology: General, 113(4), 541-555.

Johnstone, T., \& Shanks, D. R. (2001). Abstractionist and processing accounts of implicit learning. Cognitive Psychology, 42(1), 61-112.

Kinder, A., \& Assmann, A. (2000). Learning Artificial Grammars: No evidence for the acquisition of rules. Memory and Cognition, 28(8), 1321-1332. 
Knowlton, B. J., \& Squire, L. R. (1994). The information acquired during artificial grammar learning. Journal of Experimental Psychology: Learning, Memory, and Cognition, 20(1), 79-91.

Knowlton, B. J., \& Squire, L. R. (1996). Artificial grammar learning depends on implicit acquisition of both abstract and exemplar-specific information. Journal of Experimental Psychology: Learning, Memory, and Cognition, 22(1), 169-181.

Lotz, A., \& Kinder, A. (2006). Transfer in Artificial Grammar Learning: The Role of Repetition Information. Journal of Experimental Psychology: Learning, Memory, and Cognition, 32(4), 707-715.

Lubow, R. E., \& Moore, A. U. (1959). Latent Inhibition - the Effect of Nonreinforced PreExposure to the Conditional Stimulus. Journal of Comparative and Physiological Psychology, 52(4), 415-419.

Mackintosh, N. J., Kaye, H., \& Bennett, C. H. (1991). Perceptual learning in flavour aversion conditioning. Quarterly Journal of Experimental Psychology, 43(B), 297-322.

McClelland, J. L., \& Rumelhart, D. E. (1986). Parallel distributed processing: Explorations in the microstructure of cognition. (Vol. 2). Cambridge, MA: MIT Press.

McLaren, I. P. L., \& Mackintosh, N. J. (2000). An elemental model of associative learning: I. Latent inhibition and perceptual learning. Animal Learning \& Behavior, 28(3), 211246.

Navarro, J. I., Hallam, S. C., Matzel, L. D., \& Miller, R. R. (1989). Superconditioning and Overshadowing. Learning and Motivation, 20(2), 130-152.

Perruchet, P., \& Pacteau, C. (1990). Synthetic grammar learning: Implicit rule abstraction or explicit fragmentary knowledge? Journal of Experimental Psychology: General, $119(3), 264-275$. 
Pothos, E. M. (2007). Theories of Artificial Grammar Learning. Psychological Bulletin, 133(2), 227-244.

Reber, A. S. (1969). Transfer of syntactic structure in synthetic languages. Journal of Experimental Psychology, 81(1), 115-119.

Reber, A. S. (1989). Implicit learning and tacit knowledge. Journal of Experimental Psychology: General, 118(3), 219-235.

Scott, R. B., \& Dienes, Z. (2008). The conscious, the unconscious, and familiarity. Journal of Experimental Psychology-Learning Memory and Cognition, 34(5), 1264-1288.

Scott, R. B., \& Dienes, Z. (in press-a). Fluency does not express implicit knowledge of artificial grammars. Cognition (2009), doi:10.1016/j.cognition.2009.10.010.

Scott, R. B., \& Dienes, Z. (in press-b). The metacognitive role of familiarity in artificial grammar learning: transitions from unconscious to conscious knowledge. To appear. In A. Efklides \& P. Misailidi (Eds.), Trends and Prospects in Metacognition Research: Springer, US.

Servan-Schreiber, E., \& Anderson, J. R. (1990). Learning artificial grammars with competitive chunking. Journal of Experimental Psychology: Learning, Memory, and Cognition, 16(4), 592-608.

Servan Schreiber, E., \& Anderson, J. R. (1990). Learning artificial grammars with competitive chunking. Journal of Experimental Psychology: Learning, Memory, and Cognition, 16(4), 592-608.

Tunney, R. J., \& Altmann, G. T. M. (2001). Two modes of transfer in artificial grammar learning. Journal of Experimental Psychology: Learning, Memory, and Cognition, 27(3), 614-639. 
Vokey, J. R., \& Brooks, L. R. (1992). Salience of item knowledge in learning artificial grammars. Journal of Experimental Psychology: Learning, Memory, and Cognition, $18(2), 328-344$.

Vokey, J. R., \& Higham, P. A. (2005). Abstract Analogies and Positive Transfer in Artificial Grammar Learning. Canadian Journal of Experimental Psychology, 59(1), 54-61.

Zizak, D. M., \& Reber, A. S. (2004). Implicit preferences: The role(s) of familiarity in the structural mere exposure effect. Consciousness and Cognition, 13(2), 336-362. 
Appendix

Table A1

The grammar strings used, illustrated using letters.

\begin{tabular}{|c|c|c|c|c|}
\hline \multirow[b]{2}{*}{ Length } & \multicolumn{2}{|c|}{ Train } & \multicolumn{2}{|c|}{ Test } \\
\hline & Grammar A & Grammar B & Grammar A & Grammar B \\
\hline 7 & XMXRTVM & VVTRMTM & XMMXRVM & VTRRRRM \\
\hline 7 & VVTRVTM & VVTTRXM & XXRTVTM & VVRXRRM \\
\hline 7 & VTVTRVM & VVRMTRM & XXRTTVM & XMVRXRM \\
\hline 7 & VTTTTVM & XMTRRRM & ХММMMХМ & XXRRRRM \\
\hline 8 & VTTVTRVM & XMVTTRXM & VVTRTTVM & XMVTRXRM \\
\hline 8 & VVTRTVTM & VVTTRXRM & XMMXRTVM & VVTTRMTM \\
\hline 8 & VTTTTVTM & VVTRXRRM & VTTTTTVM & VVRMTRRM \\
\hline 8 & XXRVTRVM & VVRMVRXM & XMXRTVTM & XMVTRMTM \\
\hline 8 & VTVTRTVM & XMVRXRRM & XMXRTTVM & XMVRMTRM \\
\hline 8 & XMMMMMXM & VTRRRRRM & XXRTTTVM & VVTTTRXM \\
\hline 9 & XXRTVTRVM & XMVRXRRRM & XXRVTRVTM & VVRMTRRRM \\
\hline 9 & VTVTRTTVM & VVRMVTRXM & XMXRTTTVM & VVRMVRMTM \\
\hline 9 & XMMMXRVTM & VVTTTRMTM & VVTRTTVTM & XMVRMVRXM \\
\hline 9 & XMMMMXRVM & XMVTRXRRM & VTTVTRTVM & VVTTRXRRM \\
\hline 9 & XXRVTRTVM & XXRRRRRRM & XMXRVTRVM & VVTTTRXRM \\
\hline 9 & XMMMXRTVM & VVTTRMTRM & VVTRTTTVM & XMVTTRXRM \\
\hline
\end{tabular}

Note. The grammar strings were presented as strings of symbols not letters. The mapping between the letters shown above and the symbols which were actually presented is given in Table A2. 
Table A2

The mapping between the letters used to illustrate the grammar strings (shown in Table Al) and the actual symbols used in the experiments.

\begin{tabular}{lllllll}
\hline $\begin{array}{l}\text { Letters used to illustrate the grammar strings } \\
\text { in Table A1. }\end{array}$ & $\mathbf{M}$ & $\mathbf{R}$ & $\mathbf{T}$ & $\mathbf{V}$ & $\mathbf{X}$ \\
\hline $\begin{array}{l}\text { Corresponding symbols used in the } \\
\text { experiments. }\end{array}$ & & & & & & 4 \\
\hline
\end{tabular}

